Published in Operations Research Letters, vol. 22, no. 2-3,

pp. 55-62, which should be cited to refer to this work.

DOI : $10.1016 /$ S0167-6377(98)00004-2

\title{
Heuristic Solution of the Multisource Weber Problem as a $p$-Median Problem
}

Pierre HANSEN ${ }^{1}$ and Nenad MLADENOVIĆ

GERAD and École des Hautes Études Commerciales

3000, chemin de la Côte-Sainte-Catherine, Montréal, (Quebec) Canada H3T 2A7.

Éric TAILLARD

IDSIA, Corso Elvezia 36,

CH-6900 Lugano, Switzerland.

\begin{abstract}
Good heuristic solutions for large Multisource Weber problems can be obtained by solving related $p$-median problems in which potential locations of the facilities are users locations and then solving Weber problems for the sets of users of each facility.
\end{abstract}

Keywords. Location; Continuous; Heuristic.

Weber's problem is to locate a facility in the Euclidean plane in order to minimize the sum of its (weighted) distances to the locations of a given set of users. It is central to continuous location theory, and has been extended in many ways, see Wesolowsky (1993) for a recent survey. Weiszfeld's (1937) iterative algorithm, extended to ensure convergence even if an iterate coincides with a user's location (e.g. Brimberg and Love (1993)), is the standard way to solve Weber's problem. Rosen and Xue (1991) suggest accelerations. Considering simultaneous location of several facilities leads to two generalizations: (i) the Multifacility Weber problem in which several facilities each producing a different product (or rendering a different service) are to be located in order to minimize the sum of weighted distances between all facilities and all users as well as between the facilities, and (ii) the Multisource Weber problem in which several facilities producing the same product are to be located in order to minimize the sum of weighted distances from all users to their closest facility. While the former problem is one of convex optimization for which practically efficient algorithms (e.g. Calamai and Conn (1980), Overton (1983)) and a polynomial one (Xue, Rosen and Pardalos (1996)) are available, the later one is NP-hard (Megiddo and Supowit (1984)). Exact algorithms for its solution are based on branch-and-bound (Kuenne and Soland (1972), Ostresh (1973b), (1973-c), partitioning the users space (Ostresh (1973-a), (1975), Rosing (1992)), d.-c. programming (Chen, Hansen, Jaumard and Tuy (1992)) and column generation (Hansen, Jaumard, Krau and du Merle (1997)). Except for the case of two facilities, which can be solved with 10000 users (Chen, et. al. (1992)), large problems, are hard to solve exactly. Starting with Cooper's work (Cooper (1963), (1964)) of which the

\footnotetext{
${ }^{1}$ Corresponding author
} 
alternate location - allocation algorithm (ALT) is best known, many heuristics for the Multisource Weber problem have been proposed, see e.g. Chen et al. (1992) for a short survey and discussion. Recently, Bongartz, Calamai and Conn (1994) proposed a projection method (MPROJ) for the Multisource Weber problem (with any $\ell_{r}$ norm for distance). This method relaxes the $\{0,1\}$ constraints on the allocations, and solves the problem in $\mathbb{R}^{2 n+p n-p}$ space, where $n$ and $p$ denote the numbers of users and of facilities respectively. Exploiting the specific structure of the problem obtained in that way, simple projection formulas on subspaces of a domain are derived (instead of solving the system of equations in general), and used in finding descent directions (in the framework of active set methods for nonlinear programming). MPROJ is a stable algorithm that iterates until convergence is obtained. Under mild conditions, it finds local minima with integer allocation variables and exibits quadratic convergence. Descending a given number of times from randomly generated solutions, or from solutions obtained by partitioning users in sets of succesive ones along a traveling salesman tour, provides a good solution. Bongartz et al. (1994) compare MPROJ with four heuristics from the literature. As it outperforms them, it may be considered state-of-the-art.

The purpose of the present paper is (i) to revive and make operational a forgotten heuristic of Cooper (1963) for the Multisource Weber problem, and (ii) to compare it experimentally with MPROJ and with the multistart alternate location-allocation heuristic (MALT). To this effect, the similarity between the Multisource Weber problem and its discrete counterpart, i.e., the $p$-median problem, is exploited. The Multisource Weber problem can be formulated as follows (Kuenne and Soland (1972)):

$$
\min _{x_{i}, y_{i}, z_{i j}} \sum_{i=1}^{p} \sum_{j=1}^{n} z_{i j} \cdot w_{j} \cdot d_{j}\left(x_{i}, y_{i}\right)
$$

subject to

$$
\begin{gathered}
\sum_{i=1}^{p} z_{i j}=1, \quad j=1,2, \ldots, n, \\
z_{i j} \in[0,1] \quad i=1,2, \ldots, p ; j=1,2, \ldots, n,
\end{gathered}
$$

where $p$ facilities must be located to satisfy the demand of $n$ users, $x_{i}, y_{i}$ denote the coordinates of the $i^{\text {th }}$ facility, $d_{j}$ the Euclidean distance from $\left(x_{i}, y_{i}\right)$ to the $j^{\text {th }}$ user, $w_{j}$ the demand (or weight) of the $j^{\text {th }}$ user and $z_{i j}$ the fraction of this demand which is satisfied from the $i^{\text {th }}$ facility. It is easy to see that there is always an optimal solution with all $z_{i j} \in\{0,1\}$, i.e., each user satisfied from a single facility.

In the $p$-Median problem facilities must be located among a given set of sites. Otherwise the problem is the same as MW. Using similar notation to above the $p$ Median problem can be written

$$
\min _{t_{i}, z_{i j}} \sum_{i=1}^{q} \sum_{j=1}^{n} z_{i j} \cdot w_{j} \cdot d_{i j}
$$


subject to

$$
\begin{gathered}
\sum_{i=1}^{q} z_{i j}=1, \quad j=1,2, \ldots, n, \\
z_{i j} \leq t_{i}, \quad i=1,2, \ldots, q ; j=1,2, \ldots, n, \\
\sum_{i=1}^{q} t_{i}=p, \\
t_{i} \in\{0,1\} \quad i=1,2, \ldots, q, \\
z_{i j} \in\{0,1\} \quad i=1,2, \ldots, q ; j=1,2, \ldots, n .
\end{gathered}
$$

where $q>p$ sites are considered for location of $p$ facilities, $t_{i}=1$ if a facility is located at site $i, t_{i}=0$ otherwise and $d_{i j}$ denotes the distance between site $i$ and user $j$.

\begin{tabular}{r|rrrrrrrrrrrrrrrrrrr}
$p$ & 2 & 3 & 4 & 5 & 6 & 7 & 8 & 9 & 10 & 11 & 12 & 13 & 14 & 15 & 20 & 25 & 40 & 45 & 50 \\
\hline$\#$ & 1 & 2 & 4 & 4 & 5 & 5 & 6 & 7 & 7 & 3 & 6 & 7 & 9 & 11 & 15 & 19 & 30 & 35 & 41
\end{tabular}

Table 1. Number of facilities in the optimal solution of MW that coincide with users locations for the 287 users problem of Bongartz et al., (1994).

In the limit for $q$ going to infinity and a uniform distribution of sites solution of both problems coincide as any location in the plane is arbitrarily close to a site. But even for $q$ moderate the relationship between both problems can be exploited. Quite often some of the locations chosen for a facility in the Multisource Weber problem coincide with users locations (see Table 1). Sufficient conditions for this to happen in the case $p=1$, i.e., the usual Weber problem, are well-known (see e.g. Love, Morris and Wesolowsky (1988), Chapter 7). This suggests the following heuristic.

Heuristic MWPM (Multisource Weber as $p$-Median)

1. Define and solve a $p$-Median problem (PM) with the same users and demands as (MW) and the set of users locations of (MW) as potential sites for locating facilities. Let $\left(t_{i}^{*}\right)\left(z_{i j}^{*}\right)$ be the optimal solution.

2. Let $I=\left\{i \mid t_{i}^{*}=1\right\}$ and $C_{i}=\left\{j \mid z_{i j}^{*}=1\right\}$ for $i \in I$. For each $i \in I$, solve the Weber problem with users set $C_{i}$. Let $\left(x_{i}^{*}, y_{i}^{*}\right)$ be the optimal solution and $f_{i}^{*}$ its value.

3. A heuristic solution for (MW) is given by $\left\{\left(x_{i}^{*}, y_{i}^{*}\right) \mid i \in I\right\}$ and $\left(z_{i j}^{*}\right)$ with value $\sum_{i \in I} f_{i}^{*}$.

The solution obtained at completion of step 3 may be or not a local optimum for ALT. Since there is no guarantee for that (see Figure 1 for a counter-example), a Step 4 could be added to MWPM where either ALT or PROJ (methods whose convergence to a local optimum has been proved) would be applied to the solution obtained in Step 3. 


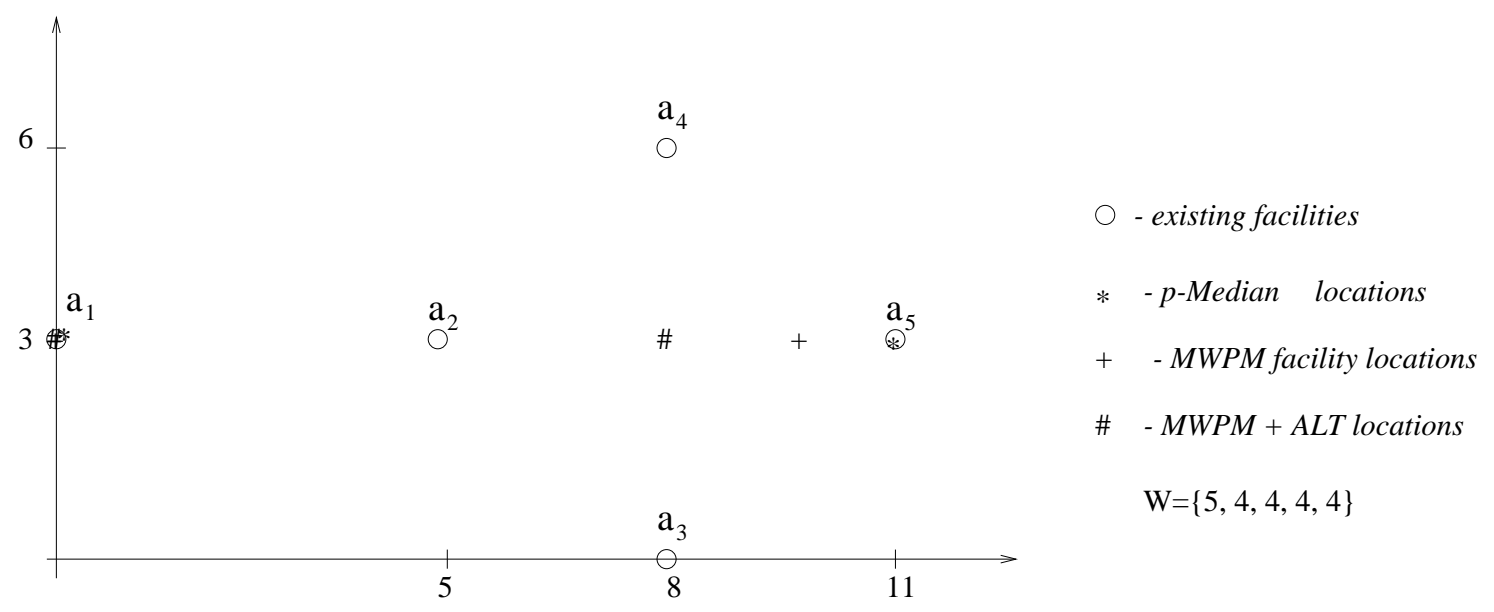

Figure 1: Counter-example where reallocations improve the MWPM solution.

Computational experience reported in Table 4 shows this improves slightly the solution of MWPM on average.

We next construct an example to illustrate this improvement of the MWPM solution. Coordinates of $n=5$ users are given as follows: $a_{1}=(0,3), a_{2}=(5,3)$, $a_{3}=(8,0), a_{4}=(8,6)$ and $a_{5}=(11,3)$. Their weights are $w_{1}=5, w_{2}=4, w_{3}=4$, $w_{4}=4$ and $w_{5}=4$. We want to locate $p=2$ facilities. Optimal $p$-Median locations are at users locations $a_{1}$ and $a_{5}$ and the corresponding objective function value is $f_{P M}=20+24 \sqrt{2}=53.9$ (Step 1 of MWPM). This solution defines a partition of the users set into two subsets: $\left\{a_{1}, a_{2}\right\}$ and $\left\{a_{3}, a_{4}, a_{5}\right\}$. In order to get the continuous solution, single facility Weber problems are solved for each users set (Step 2 of MWPM). Then the facilities have coordinates $(0,3)$ and $(9.7,3)$ with objective value $f_{M W P M}=52.8$. However, if we continue with ALT, the user located at $a_{2}=(5,3)$ will change the facility he patronizes because now the point $(9.7,3)$ is closer to him than $(0,3)$. This reallocation followed by another location step leads to the optimal solution of the problem: $(0,3)$ and $(8,3)$ with $f_{\text {opt }}=48$.

While the $p$-Median problem is NP-hard (Kariv and Hakimi (1969)), as is the Multisource Weber problem, it appears that the former is easier to solve exactly than the latter. We use for that purpose the very efficient code of Hanjoul and Peeters (1985). A simple code for Weiszfeld's algorithm is used for the less time consuming Step 2.

As mentioned above, heuristic MWPM is close to some ideas of Cooper $(1963,1964)$. Indeed, among several proposals, of which only ALT seems to have been explored in detail, Cooper $(1963,1964)$ suggests to consider in turn all $p$-tuples of users points as locations for the facilities. For each such $p$-tuple, users are assigned to the (or a) closest facility and the $p$-tuple for which the weighted sum of distances is smallest is kept. Weber problems are then solved for each facility's users set. As the number of $p$-tuples of users points may be very large, Cooper (1964) also suggests to draw a few 


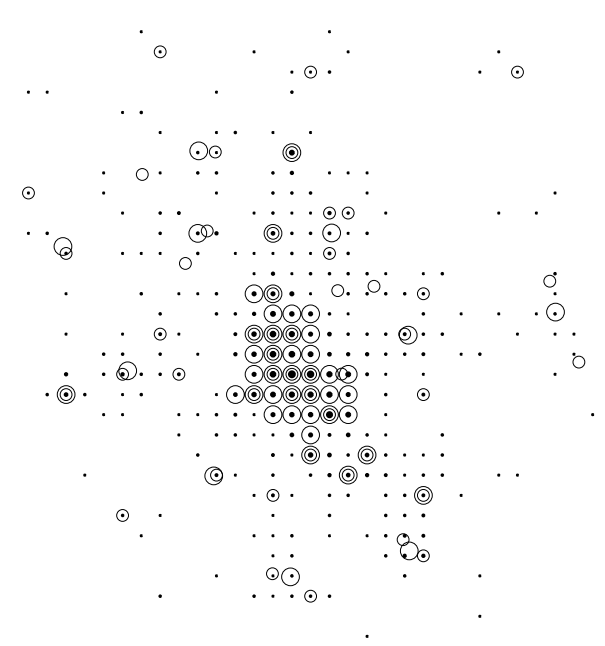

Figure 2: 287 users problem with $p=50$ facilities

of them at random and keep the best solution found. Heuristic MWPM makes Cooper's (1963, 1964) idea operational by identifying its first step as a $p$-median problem and using an efficient exact algorithm to solve it.

In the ALT heuristic $p$ points are chosen as locations of the facilities, users assigned to a closest facility and Weber problems solved for each facility's set of users. Then the procedure is iterated with the new locations of the facilities until no more changes in assigments occur. In the multistart version (MALT) of this heuristic the procedure is repeated a given number of times and the best solution kept. Cooper (1963) (1964) suggests choosing initial points among users locations and we do the same. Bongartz et al. (1994) generate two different sets of starting points: TSP (traveling salesman problem) and random location starting points. We use only the first, faster option. In our first series of experiments the number of repetitions for MPROJ and for MALT is the same, i.e. $10 p$ for $p \leq 10$ and 100 otherwise.

Results for two series of test problems are given in Table 2. The first series, due to Bongartz et al. (1994) have 287 users, corresponding to blocks of a city and $p=2$ to 50 (not all values are reported to save space). ${ }^{2}$ It appears that

\footnotetext{
${ }^{2}$ There are two typos in the data of this problem as published in Mathematical Programming 66 (1994) page 308: user 39 located at $(22,25)$ has a weight of 1 and not 6 ; user 273 is located at $(22,45)$ and not at $(22,44)$. Data for this problem and the next one are also available at http://www.idsia.ch/ eric.
} 


\begin{tabular}{|c|c|c|c|c|c|c|c|c|c|c|}
\hline \multirow[b]{2}{*}{$n$} & \multirow[b]{2}{*}{$p$} & \multicolumn{3}{|c|}{ 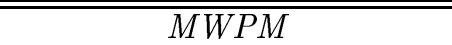 } & \multicolumn{3}{|c|}{ 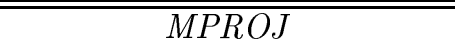 } & \multicolumn{3}{|c|}{ 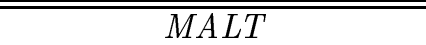 } \\
\hline & & $f_{\text {opt }}$ & Time & \%Error & $f_{o p t}$ & Time & \%Error & $f_{\text {opt }}$ & Time & \%Error \\
\hline \multirow[t]{21}{*}{287} & 2 & 14487.84 & 4.5 & 0.42 & 14427.59 & 11.1 & 0.00 & 14427.59 & 5.4 & $\overline{0.00}$ \\
\hline & 3 & 12100.37 & 4.1 & 0.04 & 12095.44 & 24.3 & 0.00 & 12106.70 & 9.6 & 0.09 \\
\hline & 4 & 10662.19 & 4.3 & 0.01 & 10661.48 & 44.5 & 0.00 & 10661.48 & 10.8 & 0.00 \\
\hline & 5 & 9716.51 & 4.5 & 0.01 & 9715.63 & 64.7 & 0.00 & 9715.63 & 13.1 & 0.00 \\
\hline & 6 & 8789.78 & 3.2 & 0.00 & 9082.11 & 88.3 & 3.33 & 8942.18 & 16.2 & 1.73 \\
\hline & 7 & 8165.18 & 4.4 & 0.00 & 8337.81 & 117.0 & 2.11 & 8284.00 & 16.8 & 1.46 \\
\hline & 8 & 7606.83 & 4.3 & 0.00 & 7796.16 & 151.0 & 2.49 & 7806.53 & 18.1 & 2.63 \\
\hline & 9 & 7129.45 & 4.2 & 0.00 & 7401.01 & 200.2 & 3.81 & 7432.64 & 19.7 & 4.25 \\
\hline & 10 & 6753.51 & 5.3 & 0.00 & 6861.23 & 249.4 & 1.60 & 7090.52 & 22.3 & 4.99 \\
\hline & 11 & 6388.77 & 4.5 & 0.00 & 6797.95 & 273.3 & 6.40 & 6729.17 & 29.9 & 5.33 \\
\hline & 12 & 6059.52 & 4.9 & 0.00 & 6381.19 & 309.0 & 5.31 & 6502.30 & 31.4 & 7.31 \\
\hline & 13 & 5764.64 & 4.1 & 0.00 & 6456.26 & 315.2 & 12.00 & 6187.75 & 29.1 & 7.34 \\
\hline & 14 & 5492.64 & 4.8 & 0.00 & 6120.85 & 359.8 & 11.44 & 6058.27 & 30.5 & 10.30 \\
\hline & 15 & 5233.05 & 4.8 & 0.00 & 5977.99 & 389.6 & 14.24 & 5767.87 & 22.6 & 10.22 \\
\hline & 20 & 4158.97 & 4.9 & 0.00 & 5061.83 & 553.4 & 21.71 & 5036.02 & 23.5 & 21.09 \\
\hline & 25 & 3349.27 & 4.6 & 0.00 & 4453.77 & 695.9 & 32.98 & 4453.76 & 24.3 & 32.98 \\
\hline & 30 & 2718.66 & 4.9 & 0.00 & 3989.97 & 819.0 & 46.76 & 3977.34 & 24.8 & 46.30 \\
\hline & 35 & 2238.58 & 4.7 & 0.00 & 3506.79 & 925.6 & 56.65 & 3614.73 & 24.7 & 61.47 \\
\hline & 40 & 1902.64 & 5.5 & 0.00 & 3380.22 & 1054.6 & 77.66 & 3367.19 & 25.7 & 76.97 \\
\hline & 45 & 1632.57 & 4.4 & 0.00 & 3157.85 & 1219.2 & 93.43 & 3096.49 & 25.1 & 89.67 \\
\hline & 50 & 1402.77 & 5.2 & 0.00 & 3025.35 & 1397.8 & 115.67 & 2854.02 & 26.9 & 103.46 \\
\hline \multicolumn{4}{|c|}{ Total error } & 0.48 & & & 507.57 & & & 487.58 \\
\hline \multirow[t]{21}{*}{654} & 2 & 815314.05 & 66.4 & 0.00 & 815313.30 & 28.7 & 0.00 & 815313.30 & 9.0 & $\overline{0.00}$ \\
\hline & 3 & 552155.98 & 75.3 & 0.20 & 551062.88 & 54.9 & 0.00 & 551062.88 & 11.6 & 0.00 \\
\hline & 4 & 288191.45 & 50.2 & 0.00 & 288190.99 & 93.1 & 0.00 & 288190.99 & 15.5 & 0.00 \\
\hline & 5 & 209068.85 & 66.7 & 0.00 & 209068.79 & 151.1 & 0.00 & 209068.79 & 23.1 & 0.00 \\
\hline & 6 & 180488.26 & 72.1 & 0.00 & 180488.21 & 259.9 & 0.00 & 180664.38 & 24.8 & 0.10 \\
\hline & 7 & 163704.21 & 62.1 & 0.00 & 163704.17 & 295.1 & 0.00 & 163704.28 & 29.5 & 0.00 \\
\hline & 8 & 147050.83 & 79.2 & 0.00 & 147050.79 & 408.9 & 0.00 & 147104.71 & 32.0 & 0.04 \\
\hline & 9 & 130936.16 & 71.3 & 0.00 & 130936.12 & 526.8 & 0.00 & 131080.02 & 36.8 & 0.11 \\
\hline & 10 & 115384.68 & 75.4 & 0.04 & 115339.03 & 641.7 & 0.00 & 116580.42 & 43.0 & 1.08 \\
\hline & 11 & 100202.58 & 65.7 & 0.07 & 100133.20 & 763.8 & 0.00 & 107229.76 & 50.9 & 7.09 \\
\hline & 12 & 94152.08 & 96.5 & 0.00 & 94152.05 & 699.4 & 0.00 & 97613.88 & 55.8 & 3.68 \\
\hline & 13 & 89466.30 & 259.3 & 0.00 & 90850.39 & 704.1 & 1.55 & 90487.69 & 51.6 & 1.14 \\
\hline & 14 & 84819.21 & 277.9 & 0.00 & 86233.92 & 623.9 & 1.67 & 86353.53 & 51.4 & 1.81 \\
\hline & 15 & 80188.58 & 196.1 & 0.00 & 81584.70 & 366.5 & 1.74 & 82431.11 & 38.3 & 2.80 \\
\hline & 20 & 63426.09 & 1357.9 & 0.00 & 68339.32 & 973.6 & 7.75 & 66384.60 & 42.1 & 4.66 \\
\hline & 25 & 52274.98 & 1420.2 & 0.00 & 58640.01 & 1317.3 & 12.18 & 57945.67 & 46.8 & 10.85 \\
\hline & 30 & 44802.81 & 4222.4 & 0.00 & 49962.54 & 1624.7 & 11.52 & 51474.53 & 52.0 & 14.89 \\
\hline & 35 & 39404.87 & 1607.8 & 0.00 & 46825.08 & 1986.6 & 18.83 & 45534.34 & 56.9 & 15.56 \\
\hline & 40 & 35904.71 & 1762.6 & 0.00 & 44698.22 & 2144.0 & 24.49 & 42355.87 & 60.2 & 17.97 \\
\hline & 45 & 32467.50 & 1296.3 & 0.00 & 40755.25 & 2448.4 & 25.53 & 39584.92 & 64.6 & 21.92 \\
\hline & 50 & 29462.98 & 2486.6 & 0.00 & 38925.55 & 2722.8 & 32.12 & 36321.78 & 71.1 & 23.28 \\
\hline \multicolumn{4}{|c|}{ Total err } & 0.31 & & & 137.36 & & & 126.96 \\
\hline
\end{tabular}

Table 2. Computational results with heuristics MWPM, MPROJ and MALT.

(i) for $p=2$ to 5 , MPROJ and, with one exception, MALT give slightly better results than MWPM, the difference being very small except for the case $p=2$; 
(ii) for larger values of $p$ MWPM gives better results than MPROJ and MALT and the magnitude of the difference steadily increases. For $p=50$ the solution of MWPM (represented by large circles in Figure 2 where dots represent demand points and their size increases with their weights) has a value less than half that of MPROJ (represented by small circles on that figure, and where facilities appear to be too dispersed);

(iii) computing times of MWPM are from 2 to 300 times smaller than those of MPROJ and from 1.2 to 7 times smaller than those of MALT.

The second series of test problems come from the Euclidean traveling salesman problem instance p654 of the TSPLIB library. They involve $n=654$ users with unit weights and $2 \leq p \leq 50$. Results are in agreement with those of the first series except that exact solution of the $p$-median problem is now time consuming, hence computing times for MWPM and MPROJ are now comparable and larger than those of MALT.

\begin{tabular}{|c|c|c|c|c|c|c|c|c|c|}
\hline \multirow[b]{2}{*}{$p$} & \multicolumn{3}{|c|}{ MWPM } & \multicolumn{6}{|c|}{ MALT } \\
\hline & $f_{\text {opt }}$ & Time & \%Error & $f_{o p t}$ & Time & $\%$ Error & $f_{\text {opt }}$ & Time & $\%$ Error \\
\hline 2 & 815314.06 & 66.4 & 0.00 & 815313.31 & 66.4 & 0.00 & 815313.31 & 664.4 & $\overline{0.00}$ \\
\hline 3 & 552156.00 & 75.4 & 0.20 & 551062.88 & 75.4 & 0.00 & 551062.88 & 754.0 & 0.00 \\
\hline 4 & 288191.44 & 50.3 & 0.00 & 288191.00 & 50.3 & 0.00 & 288191.00 & 503.4 & 0.00 \\
\hline 5 & 209068.84 & 66.3 & 0.00 & 209068.80 & 66.3 & 0.00 & 209068.80 & 663.3 & 0.00 \\
\hline 6 & 180488.27 & 72.4 & 0.00 & 180488.20 & 72.4 & 0.00 & 180488.20 & 724.1 & 0.00 \\
\hline 7 & 163704.20 & 62.3 & 0.00 & 163704.17 & 62.3 & 0.00 & 163704.17 & 623.1 & 0.00 \\
\hline 8 & 147050.83 & 79.6 & 0.00 & 147050.84 & 79.6 & 0.00 & 147050.80 & 795.7 & 0.00 \\
\hline 9 & 130936.16 & 71.3 & 0.00 & 131046.12 & 71.3 & 0.08 & 130936.12 & 713.5 & 0.00 \\
\hline 10 & 115384.68 & 75.3 & 0.02 & 115467.24 & 75.3 & 0.09 & 115357.72 & 752.5 & 0.00 \\
\hline 11 & 100202.58 & 65.6 & 0.07 & 105194.92 & 65.6 & 5.05 & 100133.20 & 655.5 & 0.00 \\
\hline 12 & 94152.08 & 96.3 & 0.00 & 95504.19 & 96.3 & 1.44 & 94152.05 & 963.0 & 0.00 \\
\hline 13 & 89466.30 & 259.2 & 0.00 & 89946.19 & 259.2 & 0.54 & 89462.98 & 2591.8 & 0.00 \\
\hline 14 & 84819.21 & 277.4 & 0.00 & 85007.74 & 277.4 & 0.22 & 84835.66 & 2773.7 & 0.02 \\
\hline 15 & 80188.58 & 196.3 & 0.00 & 80903.62 & 196.3 & 0.89 & 80221.93 & 1962.6 & 0.04 \\
\hline 20 & 63426.09 & 1357.2 & 0.02 & 64160.14 & 1357.2 & 1.18 & 63413.32 & 13572.0 & 0.00 \\
\hline 25 & 52274.98 & 1420.3 & 0.00 & 55060.60 & 1420.3 & 5.33 & 53328.73 & 14203.3 & 2.02 \\
\hline 30 & 44802.81 & 4222.2 & 0.00 & 48059.30 & 4222.2 & 7.27 & 47463.00 & 42222.4 & 5.94 \\
\hline 35 & 39404.87 & 1607.2 & 0.00 & 43988.25 & 1607.2 & 11.63 & 43230.32 & 16072.1 & 9.71 \\
\hline 40 & 35904.71 & 1762.2 & 0.00 & 39918.80 & 1762.2 & 11.18 & 38075.75 & 17622.5 & 6.05 \\
\hline 45 & 32467.50 & 1296.4 & 0.00 & 36843.35 & 1296.4 & 13.48 & 36060.48 & 12963.6 & 11.07 \\
\hline 50 & 29462.98 & 2486.3 & 0.00 & 34207.56 & 2486.3 & 16.10 & 33408.68 & 24863.1 & 13.39 \\
\hline \multicolumn{2}{|c|}{ Total error } & & 0.32 & & & 74.49 & & & 48.23 \\
\hline
\end{tabular}

Table 3. Computational results with heuristics MWPM and MALT on 654 users problem within the same and with 10 times more CPU time.

In the next table we compare MWPM and MALT on 654 users problem with the same CPU time as a stopping rule, since differences in CPU times between them (reported in Table 2) are large. It appears that the total \% error for MALT reduces to $74.49 \%$, which is still much larger than the $0.32 \%$ obtained by MWPM. The last 3 columns in Table 3 give results when the time allocated to MALT is 10 times that of MWPM. The 
total \% error drops to $48.23 \%$, which is still large. This result indicates the presence of the so-called central limit catastrophy (Baum, 1985), already observed in multi start approaches to other combinatorial optimization problems (see Boese et al. for the traveling salesman and graph bisection problems and Hansen and Mladenović (1997) for the $p$-median problem).

\begin{tabular}{|c|c|c|c|c|c|c|c|c|c|c|c|}
\hline \multirow[b]{2}{*}{$p$} & \multirow[b]{2}{*}{$\begin{array}{l}\text { Best } \\
\text { found }\end{array}$} & \multicolumn{6}{|c|}{$\%$ DEVIATION } & \multicolumn{4}{|c|}{ CPU TIME } \\
\hline & & PM & $\begin{array}{l}\mathrm{PM+} \\
\mathrm{WSZ}\end{array}$ & $\begin{array}{l}\mathrm{PM+} \\
\mathrm{ALT}\end{array}$ & $\begin{array}{l}\text { PM+ } \\
\text { PROJ }\end{array}$ & MPROJ & MALT & $\mathrm{PM}$ & $\begin{array}{l}\text { PM+ } \\
\text { PROJ }\end{array}$ & MPROJ & MALT \\
\hline 2 & 1923.73 & 0.07 & 0.03 & 0.00 & 0.00 & 0.00 & 0.00 & 5.9 & 5.9 & 25.8 & 5.4 \\
\hline 3 & 1642.45 & 0.03 & 0.03 & 0.01 & 0.01 & 0.00 & 0.00 & 8.3 & 8.3 & 36.3 & 7.2 \\
\hline 4 & 1442.66 & 0.15 & 0.04 & 0.02 & 0.00 & 0.00 & 0.00 & 10.6 & 10.6 & 58.2 & 10.2 \\
\hline 5 & 1295.58 & 0.14 & 0.06 & 0.06 & 0.06 & 0.02 & 0.00 & 12.9 & 13.0 & 70.2 & 11.8 \\
\hline 6 & 1185.62 & 0.09 & 0.04 & 0.03 & 0.03 & 0.03 & 0.00 & 14.9 & 15.0 & 72.8 & 13.9 \\
\hline 7 & 1092.30 & 0.28 & 0.15 & 0.14 & 0.14 & 0.00 & 0.00 & 16.4 & 16.5 & 81.6 & 14.5 \\
\hline 8 & 1012.70 & 0.10 & 0.01 & 0.01 & 0.01 & 0.01 & 0.00 & 25.8 & 25.9 & 103.2 & 18.0 \\
\hline 9 & 953.44 & 0.24 & 0.09 & 0.01 & 0.01 & 0.00 & 0.00 & 23.7 & 23.8 & 118.3 & 19.3 \\
\hline 10 & 904.27 & 0.23 & 0.03 & 0.00 & 0.00 & 0.13 & 0.00 & 23.1 & 23.2 & 133.2 & 18.3 \\
\hline 11 & 858.22 & 0.27 & 0.12 & 0.03 & 0.03 & 0.01 & 0.00 & 26.4 & 26.5 & 150.0 & 19.8 \\
\hline 12 & 820.20 & 0.46 & 0.28 & 0.13 & 0.13 & 1.62 & 0.00 & 26.7 & 26.8 & 148.7 & 21.7 \\
\hline 13 & 783.85 & 0.39 & 0.08 & 0.00 & 0.00 & 0.17 & 0.31 & 29.3 & 29.5 & 169.0 & 21.3 \\
\hline 14 & 755.35 & 0.37 & 0.17 & 0.00 & 0.00 & 1.46 & 0.96 & 30.6 & 30.8 & 173.4 & 22.3 \\
\hline 15 & 725.57 & 0.63 & 0.17 & 0.00 & 0.00 & 0.60 & 1.74 & 28.5 & 28.7 & 191.7 & 23.6 \\
\hline 20 & 616.48 & 0.28 & 0.08 & 0.00 & 0.00 & 1.59 & 4.11 & 38.1 & 38.5 & 217.2 & 32.3 \\
\hline 25 & 535.86 & 0.37 & 0.02 & 0.00 & 0.00 & 3.01 & 7.45 & 43.6 & 43.9 & 313.8 & 39.3 \\
\hline 30 & 478.24 & 0.63 & 0.13 & 0.00 & 0.00 & 3.95 & 9.03 & 48.8 & 49.3 & 365.5 & 45.3 \\
\hline 35 & 434.43 & 0.46 & 0.03 & 0.00 & 0.00 & 4.53 & 12.03 & 55.7 & 56.2 & 460.7 & 49.4 \\
\hline 40 & 396.32 & 0.73 & 0.25 & 0.00 & 0.00 & 6.39 & 14.63 & 57.0 & 57.6 & 529.0 & 50.5 \\
\hline 45 & 365.21 & 0.55 & 0.11 & 0.00 & 0.00 & 8.18 & 19.29 & 57.1 & 57.9 & 587.0 & 49.6 \\
\hline 50 & 337.60 & 0.51 & 0.11 & 0.00 & 0.00 & 10.34 & 19.61 & 64.6 & 65.3 & 658.0 & 48.4 \\
\hline \multicolumn{2}{|c|}{ Total error } & 7.00 & 2.00 & 0.44 & 0.42 & 42.03 & 89.17 & & & & \\
\hline
\end{tabular}

Table 4. Computational results for the 287 users problem with unit weights.

In Table 4 we compare again heuristics on the 287 users problem, but now with unit weights (as recommended by an anonymous referee). The exact $p$-median solution method of Hanjoul and Peeters (1985), becomes very time consuming for $p \geq 14$ (in fact, computing times jump from less than 20 seconds to about 2000 seconds). So, in order to show how close the solutions of PM and multisource Weber problems are, in Step 1 of MWPM we use the multistart fast interchange heuristic method of Whitaker (1985), Hansen and Mladenovic (1997) instead of an exact algorithm. This heuristic is restarted 10 times and the best solution kept. In columns from 3 to 8 of Table 2 the $\%$ deviation relative to the best solution among methods compared are reported. In the last three columns CPU time of PM, PROJ and MALT are given. The CPU times of MWPM and PM+ALT are not reported because they are usually larger than that of PM by less than 0.05 sec. Thus, the running times of PM, MWPM and PM+ALT are almost the same. It appears that the integer solution obtained by the heuristic method (in Step 1 of MWPM) gives solutions of better quality than the state-of-the-art 
heuristics MALT and MPROJ (compare the total 7\% deviation for PM with $42 \%$ and $89 \%$ for MPROJ and MALT respectively). It appears also that the solution of PM are notably improved in Step 2, and a bit more if ALT or PROJ is used instead of the Weiszfeld (1937) procedure (which coincides with the first iteration of ALT).

Acknowledgements: Research of the first two authors was supported by Office of Naval Research Grant N00014-92-J-1194, Natural Sciences and Engineering Research Council of Canada Grant GPO 105574 and Fonds pour la Formation des Chercheurs et l'Aide à la Recherche Grant 32EQ 1048. Research of the third author was supported by an International Post-doctoral Fellowship of Natural Sciences and Engineering Research Council of Canada, Grant OGPOO 39682 and done mostly during a visit at CRT, Montreal. The authors thank Paul Calamai for making the program for his projection method written with Ingrid Bongartz and Andrew Conn, available to them, Dominique Peeters for communicating his program for the $p$-Median problem, written with Pierre Hanjoul, and an anonymous referee for his remarks and suggestions.

\section{References}

BAUM, E.B. 1986. Toward practical 'neural' computation for combinatorial optimization problems. In J. Denker (Eds.), Neural networks for computing, American Institute of Physics.

Boese, K.D., Kahng A.B., \& Muddu, S. 1994. A new adaptive multi-start technique for combinatorial global optimizations. Operations Research Letters 16, 101-113.

Brimberg, J., AND R.F. Love. 1993. Global convergence of a generalized iterative procedure for the minisum location problem with $l_{p}$ distances. Operations Research 41, 1153-1163.

Bongartz, I., P.H. Calamai And A.R. Conn 1994. A projection method for $l_{p}$ norm location-allocation problems. Mathematical Programming 66, (1994) 283-312.

Calamai, P.H. AND A.R. Conn. 1980. A stable algorithm for solving the multifacility location problem involving Euclidean distances. SIAM Journal on Scientific and Statistical Computing 1, 512-525.

Chen P.-C., P. Hansen, B. Jaumard, H. Tuy. 1992. Solution of the multisource Weber and conditional Weber problems by d.-c. programming, GERAD Research Report G-92-35, University of Montreal, Canada (to appear in Operations Research)

Cooper, L. 1963. Location-allocation problems. Operations Research 11, 301-343.

CoOper, L. 1964. Heuristic methods for location-allocation problems. SIAM Review 6, $37-53$.

Eilon, S., C. Watson-Gandy and N. Christofides. 1971. Distribution Management: Mathematical modeling and practical analysis. Griffin, London.

Hanjoul, P. And D. Peeters. 1985. A comparison of two dual-based procedures for solving the $p$-median problem. European Journal of Operational Research 20 387-396.

Hansen, P., B. Jaumard, S. Krau and O. Du Merle. 1997. A stabilized column generation algorithm for the multisource Weber problem, GERAD research report (forthcoming). 
Hansen, P. And N. Mladenović. 1997. Variable neighbourhood search for the $p$-median. GERAD research report G-97-39.

KARIV, O. AND S.L. HAKIMI. 1979. An algorithmic approach to network location problems; part 2. The p-medians. SIAM Journal on Applied Mathematics 37,539-560.

Kuenne, R. E., And R. M. Soland. 1972. Exact and approximate solutions to the multisource Weber problem. Mathematical Programming 3, 193-209.

Love, R. F., J. G. Morris And G. O. Wesolowsky. 1988. Facilities Location: Models and Methods. North-Holland, New York.

Megiddo, And K.J. Supowit. 1984. On the complexity of some common geometric location problems. SIAM Journal on Computing 13, 182-196

Ostresh JR., L.M. 1973a. TWAIN - Exact solutions to the two source location-allocation problem. in: G. Rushton, M.F. Goodchild and L.M. Ostresh Jr. (eds.), Computer Programs for Location-Allocation Problems, Monograph Number 6, Department of Geography, University of Iowa, Iowa City, IA, 15-28.

Ostresh JR., L.M. 1973b. MULTI - Exact solutions to the $M$-center location-allocation problem. in: G. Rushton, M.F. Goodchild and L.M. Ostresh Jr. (eds.), Computer Programs for Location-Allocation Problems, Monograph Number 6, Department of Geography, University of Iowa, Iowa City, 29-53.

Ostresh JR., L.M. 1973c. An investigation of the multiple location-allocation problem. $\mathrm{PhD}$ Thesis, The University of Iowa, Iowa City, IA.

Ostresh JR., L.M. 1975. An efficient algorithm for solving the two center location-allocation problem. Journal of Regional Science 15, 209-216.

Overton, M.L. 1983. A quadratically convergent method for minimizing a sum of Euclidean norms, Mathematical Programming 27, 34-63.

Rosen, J. B., And G.-L. Xue. 1991. Computational comparison of two algorithms for the Euclidean single facility location problem. ORSA Journal on Computing 3, 207-212.

Rosing, K.E. 1992. An optimal method for solving the (generalized) multi-Weber problem. European Journal of Operational Research 58, 414-426.

Weiszfeld, E. 1937. Sur le point pour lequel la somme des distances de $n$ points donnés est minimum. Tôhoku Mathematical Journal 43, 355-386.

Wesolowsky G. 1993. The Weber problem: history and perspectives. Location Science 1, $5-23$.

Whitaker, R. 1983. A fast algorithm for the greedy interchange for large-scale clustering and median location problems. INFOR 21, 95-108.

Xue, G.L., J.B. Rosen and P.M. Pardalos. 1996. A polynomial time dual algorithm for the Euclidean multifacility location problem. Operations Reserch Letters 18, 201-204. 